\title{
Reconstruction of spectral reflectance based on mixed weighting and local optimization
}

\author{
${ }^{1}$ Leihong Zhang, ${ }^{1 *}$ Runchu Xu, ${ }^{1}$ Shuangquan Lu, ${ }^{1}$ Liuhua Yang, \\ ${ }^{1}$ Xiao Yuan, ${ }^{2}$ Kaiming Wang and ${ }^{2 *}$ Dawei Zhang \\ ${ }^{1}$ College of Communication and Art Design, University of Shanghai for Science and \\ Technology, Shanghai, 200093, China, *xrc1231@163.com \\ ${ }^{2}$ School of Optical-Electrical and Computer Engineering, University of Shanghai for \\ Science and Technology, Shanghai, 200093, China.* friedrich_suse@foxmail.com
}

Received: 23.03 .2020

\begin{abstract}
High-fidelity colour reproduction can be performed through reconstructing a spectral reflectance of an object surface. The particular branches in need of this reproduction range from colour printing to artistic fields. In order to improve the reconstruction accuracy for the spectral reflectance, we suggest a spectral-reflectance reconstruction method based on a mixed weighting (MW). This method is a combination of a number of earlier techniques, namely a Wiener estimation method and so-called methods of weighting on a group with smaller colour difference and weighting on a group with smaller spectral reflectance error. Specifically, we have obtained the spectral estimation with higher reconstruction accuracy by weighting the reconstruction spectra obtained by different methods. The weights for these methods have been selected through minimizing the colour difference. The MW method makes a full use of advantages of the underlying methods. It reveals high accuracy and reduces the shortcomings of those methods. Our experimental results confirm that the MW method improves the reconstruction accuracy and the stability of spectral-reflectance data.
\end{abstract}

Keywords: colour difference, spectral reflectance error, spectral reflectance reconstruction, weighting, local optimization

UDC: 535.67

\section{Introduction}

A spectral reflectance is one of the most comprehensive and accurate ways to characterize the colour information of object surfaces. Technologies of spectral-reflectance reconstruction have many applications in such fields as skin-colour recognition [1], colour printing, textiles [2], and cell imaging [3]. Moreover, the above technologies can reduce a known phenomenon of metamerism [4-7].

Traditional spectral-reconstruction methods include mainly a pseudo-inverse (PINV) method, a Wiener estimation (WE) method, and a principal component analysis (PCA). The PINV method is simple and convenient, but its reconstruction accuracy is poor. Moreover, the technique suffers from noise [8]. The PCA can reduce spectral dimensions and the effects of noise, though the reflectance is more often prone to negative values [9, 10]. Although the reconstruction accuracy of the WE method is higher than that of the PINV method and the PCA, it has some difficulties in meeting modern demands $[11,12]$.

Many scholars have tried their best to improve the reconstruction accuracy for the spectral reflectance. In particular, any single-pixel detector provides a feasible way to improve the reconstruction accuracy of spectral images. On this route, Zhang [13] and Li et al. [14] have used 
single-pixel detectors, along with the PCA and the compressed-sensing algorithms, to reconstruct the spectral images. This reveals good enough performance in colour reproduction. In addition, selection of training samples and optimization of light sources can provide another possibility for improving the spectral-reconstruction accuracy. Liang et al. [15] have improved the accuracy through a weighted PINV method and optimal selection of training samples based on a colourspace distance. H. Otsu et al. [16] have proposed a greedy clustering algorithm to classify a set of training samples. When the class of spectral images belongs to the class used for training, the reconstruction accuracy of the spectral images is high.

Cao et al. [17] have suggested a so-called method of weighting on a group with smaller colour difference (WSCG) to improve the spectral-reconstruction accuracy. Specifically, the colour differences between a single testing sample and training samples are used as the weights to weight the spectral reflectance of the corresponding training samples. Zhang et al. [18] have developed a spectral-reflectance reconstruction method based on tristimulus values under multiple light sources by selecting local training samples. The method improves notably the spectralreconstruction accuracy. Kong et al. [19] have used a genetic algorithm to optimize selection of light sources, which also improves this accuracy.

In the recent years, learning-based approaches to improving accuracy of the reconstruction of spectral images have gradually become a focus of many researches. For instance, V. Heikkinen [20] has used Gaussian processes and a combination of kernels to construct the estimation models, which improves the reconstruction accuracy of spectral images. Koundinya et al. [21] have proposed a reconstruction method through a convolution neural network. Their reconstruction manifests good performance in terms of the average relative absolute error and the root-meansquare error (RMSE). Han et al. [22] have explored a spectral reconstruction for the spectral superresolution with an available RGB image. It yields a high-frequency content of fine spectral wavelengths in a narrow-band interval.

Basing on the studies available for the WSCG method, we should point out a number of its shortcomings. In the process of spectral-reflectance reconstruction, Cao et al. [17] have selected the same number of training samples for different testing samples, which corresponds to the average variation trend of RMSE and goodness-of-fit coefficient (GFC). This simplifies the calculations though increases the reconstruction error to some extent. To improve the reconstruction accuracy for spectral reflectance in the frame of the WSCG method, we suggest a WSCG method with a global optimal solution (WSCG-GOS). Specifically, we traverse the training dataset to select the appropriate number of training samples for different testing samples, which is based on minimizing the colour difference. It turns out that the WSCG-GOS method has good performance and accuracy in the spectral reconstruction. However, the calculation time becomes increased greatly, which is not desirable in practice. Therefore, we suggest a WSCG method with a local optimal solution (WSCG-LOS). In particular, this WSCG-LOS method selects a small number of local training samples for different testing samples, while the number of selected training samples is the same. In the local space of training-sample sets, we select the appropriate number of training samples for different testing samples by minimizing the colour difference. As a result, the WSCG-LOS method finds the optimal solution in the local space of training-sample sets, which can achieve a good balance between the calculation time and the reconstruction accuracy. Our experimental results show that the reconstruction accuracy of the WSCG-LOS method is higher than those of the WE and PINV methods. However, since the WSCG-LOS method uses the colour difference as a weight, the metamerism phenomenon still exists. 
To improve further the spectral-matching degree, we also develop a method of weighting on a group with smaller spectral-reflectance error (WSREG). Specifically, spectral-reflectance errors (SREs) are used as the weights aimed at weighting the spectral reflectances of the corresponding training samples. Moreover, we compare the reconstruction accuracies and the calculation times of the WSREG, WSREG-GOS and WSREG-LOS methods with the corresponding parameters typical for the WSCG-GOS and WSCG-LOS methods. Our experimental results reveal that the WSREG-LOS method is characterized by higher reconstruction accuracy and less calculation time. The WSREG-LOS method improves further the spectral-matching degree but the appropriate colour difference is greater than those of the WSCG-LOS and WE methods.

Keeping the shortcomings of the above methods in mind, we suggest a method of mixed weighting (MW) to improve still further the spectral-reconstruction accuracy. The spectral estimation with higher accuracies can be obtained by weighting the reconstruction spectra obtained with different methods. The MW method combines all the advantages of the underlying methods, thus improving their reconstruction accuracy and reducing their shortcomings. The experimental data obtained by us indicates that the MW method provides the spectral estimation characterized with both high accuracy and high data stability. Therefore, this method can be regarded as a useful generalization of different reconstruction methods.

\section{Traditional algorithms}

\subsection{PINV algorithm}

In the process of multi-spectral imaging, the camera response values are given by

$$
C_{K \times 1}=W_{K \times Q} R_{Q \times 1}+N_{K \times 1},
$$

where $K$ denotes the number of camera channels, $R$ the spectral reflectance, $W$ the spectral response matrix, $N$ the image noise, and $Q$ the dimension of spectra sampling. In this study, the spectrum in the $400-700 \mathrm{~nm}$ band is sampled using $10 \mathrm{~nm}$ intervals. Therefore, the spectral reflectance can be expressed as a vector with $31 \times 1$ dimensions, i.e. $Q$ is equal to 31 . The inverse $M$ of the spectral response matrix $W$ is a conversion matrix between the camera response value $V$ and the reconstructed spectral reflectance $R_{r e c}$. Here $R_{\text {rec }}$ can be expressed as

$$
R_{\text {rec }}=M V \text {. }
$$

If the spectral response matrix $W$ is singular, the conversion matrix $M_{1}$ becomes pseudoinverse. Then the conversion matrix $M_{1}$ stands as

$$
M_{1}=R V^{+}=R V^{T}\left(V V^{T}\right)^{-1}
$$

with $V^{+}$being the pseudo-inverse matrix of the camera response $V$ and $T$ denoting the transpose of this matrix.

\subsection{WE algorithm}

The main idea of the WE algorithm is to minimize the difference between the reconstructed and original spectral reflectances:

$$
E\left\{\left|R-R_{\text {rec }}\right|^{2}\right\}=E\left\{|R-M V|^{2}\right\} \rightarrow M I N
$$

According to Ref. [23], the conversion matrix $M_{2}$ used for the WE method can be calculated as

$$
M_{2}=K_{R} W^{T}\left(W K_{R} W^{T}+K_{N}\right)^{-1} .
$$

Ukr. J. Phys. Opt. 2020, Volume 21, Issue 2 
In Eq. (5), $K_{R}$ is the autocorrelation matrix of the spectral reflectance $R$ and $K_{N}$ implies the noise covariance matrix.

\section{MW method}

\subsection{Spectral-reflectance reconstruction using WSCG}

In the present study we take the CIE 1976 colour-difference equation taken from Ref. [24]. Namely, the colour difference $\Delta E_{n}$ can be found as

$$
\Delta E_{n}=\sqrt{\left(L_{\text {test }}-L_{n}\right)^{2}+\left(a_{\text {test }}-a_{n}\right)^{2}+\left(b_{\text {test }}-b_{n}\right)^{2}},
$$

where $L$ denotes the luminance, $a$ the redness-greenness axis, $b$ the yellowness-blueness axis, test the testing sample, $\Delta E_{n}$ the colour difference between a single testing sample and an $n$-th training sample.

The WSCG method reconstructs the spectral reflectance of a testing sample by using the colour differences as weights, in order to weight the spectral reflectance of the corresponding training samples. Four different weighting models are established, which can be expressed as follows:

$$
\begin{aligned}
& R=K\left(\frac{R_{1}}{\Delta E_{1}^{2}}+\frac{R_{2}}{\Delta E_{2}^{2}}+\ldots+\frac{R_{n}}{\Delta E_{n}^{2}}\right), K=1 /\left(\frac{1}{\Delta E_{1}^{2}}+\frac{1}{\Delta E_{2}^{2}}+\ldots+\frac{1}{\Delta E_{n}^{2}}\right), \\
& R=K\left(\frac{R_{1}}{\Delta E_{1}}+\frac{R_{2}}{\Delta E_{2}}+\ldots+\frac{R_{n}}{\Delta E_{n}}\right), K=1 /\left(\frac{1}{\Delta E_{1}}+\frac{1}{\Delta E_{2}}+\ldots+\frac{1}{\Delta E_{n}}\right), \\
& R=K\left(\Delta E_{n}^{2} R_{1}+\Delta E_{n-1}^{2} R_{2}+\ldots+\Delta E_{1}^{2} R_{\mathrm{n}}\right), \mathrm{K}=1 /\left(\Delta E_{1}^{2}+\Delta E_{2}^{2}+\ldots+\Delta E_{\mathrm{n}}^{2}\right), \\
& R=K\left(\Delta E_{n} R_{1}+\Delta E_{n-1} R_{2}+\ldots+\Delta E_{1} R_{n}\right), K=1 /\left(\Delta E_{1}+\Delta E_{2}+\ldots+\Delta E_{n}\right) .
\end{aligned}
$$

Here $R$ is the reconstructed spectral reflectance, $\Delta E_{1} \ldots \Delta E_{n}$ imply the colour differences in ascending order, $R_{l} \ldots R_{n}$ the spectral reflectances of the training samples, and the $K$ parameter ensures that the reflectance values lie in the range from 0 to 1 .

The trends for the RMSE and GFC changes occurring when the number of training samples varies from 1 to 10 have earlier been analyzed for the case of WSCG method [17]. To simplify calculations, the same number of the training samples has been selected for different testing samples, which increases the reconstruction error to some extent. We propose the WSCG-GOS and WSCG-LOS methods to improve the reconstruction accuracy of the WSCG. The WSCG-GOS method traverses the training dataset to reconstruct the spectrum of a single testing sample with minimized colour difference. In particular, this approach chooses the number of the training samples for different testing samples by minimizing the colour difference. The WSCG-GOS method obtains the global optimal solution for the testing samples, although the calculation time is greatly increased. Of course, this is not desirable in practice.

The WSCG-LOS method chooses the same number of the training samples for different testing samples according to the relationship between the number of the training samples and the reconstruction accuracy. Then a selected training sample is only a small part of the training datasets, and their weights are large. In the local space of training sample sets, we select the appropriate number of the training samples for different testing samples, basing on minimization of the colour difference. Then the WSCG-LOS method would imply finding an optimal solution in the local space of training datasets. This reduces notably the calculation time and still does not lower the reconstruction accuracy. 


\subsection{Spectral-reflectance reconstruction using WSREG}

Here we use the SRE to evaluate the spectral similarity. The SRE can be found as

$$
S R E=\sqrt{\frac{\sum\left(R-R_{r e c}\right)^{T}\left(R-R_{r e c}\right)}{N}},
$$

where $N$ means the spectral dimension, $R$ the spectral reflectances of the training samples, $R_{\text {rec }}$ the first reconstructed spectral reflectance based on the traditional methods, and $T$ the transpose of the matrix.

The WSREG method has been suggested to obtain the spectral estimation with a high spectral-matching degree. Specifically, the SREs between a single testing sample and the training samples are used as the parameters for weighting the spectral reflectances of the corresponding training samples. Again, the same four weighting models are employed:

$$
\begin{aligned}
& R=K\left(\frac{R_{1}}{S R E_{1}^{2}}+\frac{R_{2}}{S R E_{2}^{2}}+\ldots+\frac{R_{n}}{S R E_{n}^{2}}\right), K=1 /\left(\frac{1}{S R E_{1}^{2}}+\frac{1}{S R E_{2}^{2}}+\ldots+\frac{1}{S R E_{n}^{2}}\right), \\
& R=K\left(\frac{R_{1}}{S R E_{1}}+\frac{R_{2}}{S R E_{2}}+\ldots+\frac{R_{n}}{S R E_{n}}\right), K=1 /\left(\frac{1}{S R E_{1}}+\frac{1}{S R E_{2}}+\ldots+\frac{1}{S R E_{n}}\right), \\
& R=K\left(S R E_{n}^{2} R_{1}+S R E_{n-1}^{2} R_{2}+\ldots+S R E_{1}^{2} R_{n}\right), K=\frac{1}{\left(S R E_{1}^{2}+S R E_{2}^{2}+\ldots+S R E_{n}^{2}\right)}, \\
& R=K\left(S R E_{n} R_{1}+S R E_{n-1} R_{2}+\ldots+S R E_{1} R_{n}\right), K=\frac{1}{\left(S R E_{1}+S R E_{2}+\ldots+S R E_{n}\right)} .
\end{aligned}
$$

Here $R$ means the reconstructed spectral reflectance, $S R E_{1} \ldots S R E_{n}$ are the SREs in ascending order, $R_{l} \ldots R_{n}$ the spectral reflectances of the training samples, and $K$ ensures that the reflectance values lie between 0 and 1 .

Similar to the WSCG-GOS and WSCG-LOS methods, we also develop a WSREG-GOS method and a WSREG-LOS method. It turns out that the WSREG-LOS approach has good enough performance, high reconstruction accuracy and a less calculation time.

\subsection{Spectral-reflectance reconstruction using $M W$}

To improve further the reconstruction accuracy and overcome the shortcomings of the WE, WSCG-LOS and WSREG-LOS methods, we suggest the MW method. Specifically, higheraccuracy spectral estimations can be obtained by weighting the reconstructed spectra obtained using different methods. The spectral estimation $R$ reads as follows:

$$
R=W 1 \cdot R 1+W 2 \cdot R 2+\ldots+W n \cdot R n .
$$

In Eq. (16), $R 1, R 2, \ldots, R n$ are the spectra reconstructed with different methods and $W 1, W 2, \ldots, W n$ the corresponding weights. We remind that, in selecting the weights, the three trivial rules have to be adhered to: (1) the sum of the weights must be unit, (2) the selection range for every weight is $[0 ; 1]$, and (3) the interval of single weight is 0.1 .

The steps needed to reconstruct the spectral reflectance basing on the MW are illustrated in Fig. 1. The main steps can be described as follows.

(1) We use a spectrophotometer and a digital camera to obtain respectively the original spectral reflectance and the camera response values for the samples.

(2) We establish a smaller-colour difference group according to the camera response values for the

Ukr. J. Phys. Opt. 2020, Volume 21, Issue 2 


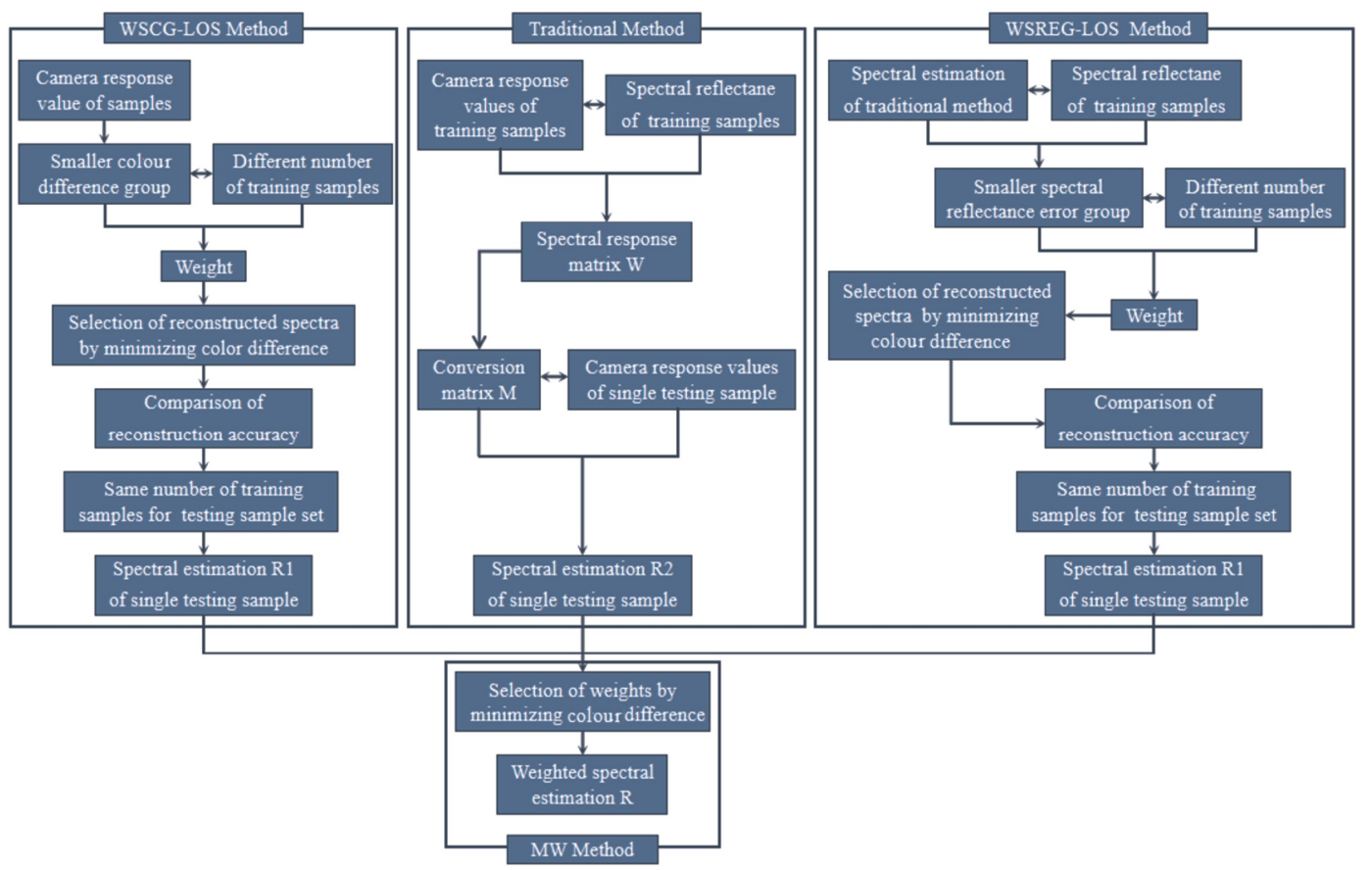

Fig. 1. Scheme of spectral-reflectance reconstruction based on the MW approach.

samples. Different numbers of training samples are selected at a certain interval, and the reconstructed spectra of the testing samples are selected by minimizing the colour difference. We select the same number of local training samples for different testing samples by exploring the relationship between the overall reconstruction accuracy and the number of training samples. One can obtain the reconstructed spectra R1 with the WSCG-LOS method, using the local training dataset and minimizing the colour difference.

(3) We use one of the traditional methods, namely the WE method, to obtain the reconstructed spectra R2.

(4) We establish the smaller-SRE group according to the first reconstructed spectra R2 for the testing dataset and the original spectra of the training dataset. Concerning the step (2), we use the WSREG-LOS method to obtain the reconstructed spectra R3.

(5) The spectra reconstructed with different methods are weighted to obtain the spectral estimation with higher accuracy, using the MW method. The weights referred to different methods can be obtained by minimizing the colour difference.

\subsection{Accuracy evaluation for the spectral-reflectance reconstruction}

We use a standard CIE 1976 colour difference to evaluate the degree of colour distortion, which is expressed by Eq. (6). The RMSE and the GFC are used to evaluate the spectral-matching degree. The specific RMSE [25-27] and GFC [28] parameters are expressed as follows:

$$
\mathrm{RMSE}=\sqrt{\frac{1}{n} \sum_{i=1}^{\mathrm{n}}\left(R_{i}-r_{i}\right)^{2}}, G F C=\frac{\left|\sum_{i} R_{i} r_{i}\right|}{\left|\sum_{i} R_{i}^{2}\right|\left|\sum_{i} r_{i}^{2}\right|},
$$

where $R_{i}$ and $r_{i}$ represent the reflectances for the original and reconstructed spectra, respectively. 


\section{Experimental studies}

The experimental steps needed for reconstructing the spectral reflectance in the frame of our MW method are illustrated in Fig. 2. These steps can be described as follows.

(1) The sample set is placed at the angle $45^{\circ}$ with respect to the lighting direction. A digital camera with pre-set parameters is used to obtain the camera-response values. The original spectral reflectance is measured by a spectrophotometer.

(2) The WSCG-LOS, WE and WSREG-LOS methods are used to obtain the appropriate reconstructed spectra R1, R2 and R3 of a single testing sample.

(3) We weight the reconstructed spectra R1, R2 and R3 using the MW method and obtain the spectral estimation. Note that the alternative PINV method is simple in calculations but sensitive to the system noise; its experimental reconstruction accuracy is poor enough.

Therefore, in order to improve the reconstruction accuracy of the WSREG-LOS and MW methods, we use the WE method and obtain experimentally the reconstructed spectra R2.

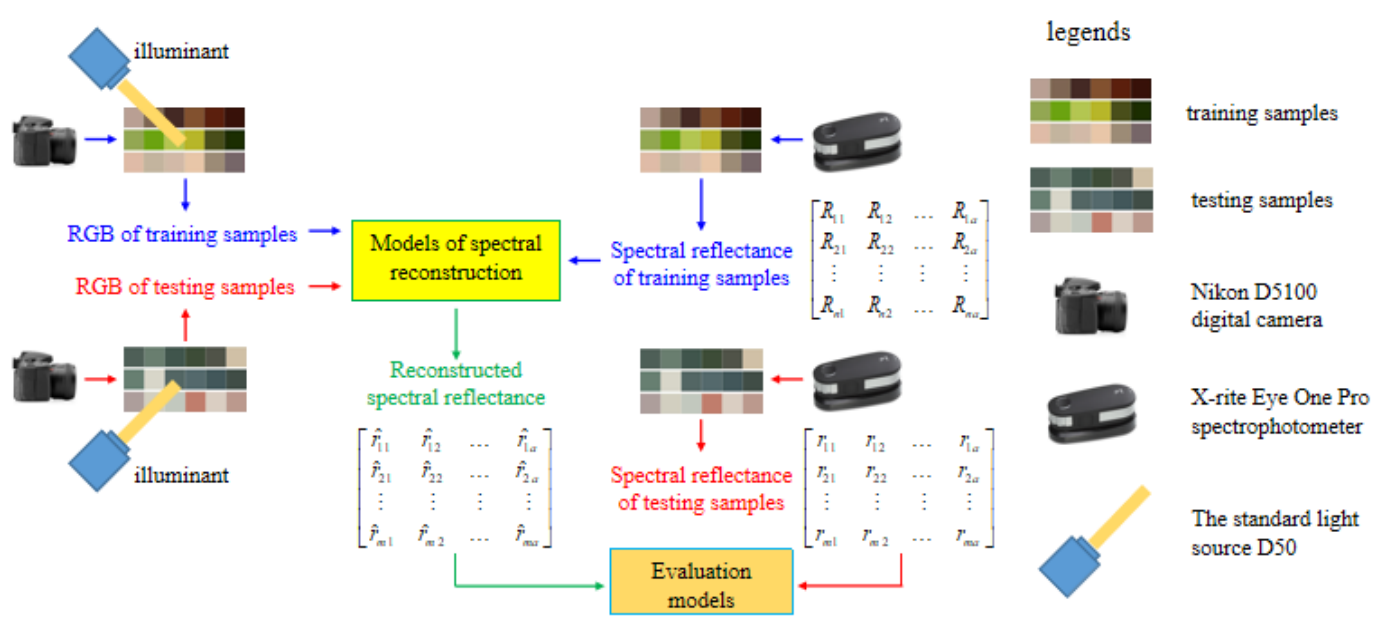

Fig. 2. Experimental stages of spectral-reflectance reconstruction.

In our experiments, the X-rite's Colour Checker Digital SG (SG140) has been selected as both a training dataset and a testing dataset. It should be noted that, in the experimental self-test, the sample of which reflectance is being reconstructed is removed from the training dataset. The spectral reflectance is measured, using an X-rite Eye One Pro spectrophotometer. The spectral range is from 400 to $700 \mathrm{~nm}$, with the intervals $10 \mathrm{~nm}$ [29, 30]. A standard light source D50 has been provided using a Judge II-S X-Rite Macbeth standard light-source lamp box. The parameters of the Nikon D5100 digital camera have been fixed during the experiment. Namely, the aperture size is F4.2, the exposure time $1 / 30 \mathrm{~s}$, the white balance is sunny, and the ISO is equal to 200 . The dark-current noise has been recorded with the camera-lens cap closed. This value has been subtracted from the camera response values [15]. In addition, we have employed the computing equipment Lenovo y7000p with 16GB DDR4 RAM, NVIDIA GTX1060 GPU and Intel i5-8300H CPU.

\subsection{Comparison of WSCG, WSCG-GOS and WSCG-LOS methods}

We have reproduced the WSCG method following the experimental procedures by Cao [17]. The main regularities observed in our experiments for the $\overline{\Delta \mathrm{E}}, \overline{\mathrm{RMSE}}$ and $\overline{\mathrm{GFC}}$ parameters are shown in Fig. 3. Here the number of training samples varies from 1 to 20. As seen from Fig. 3a,

Ukr. J. Phys. Opt. 2020, Volume 21, Issue 2 
(a)

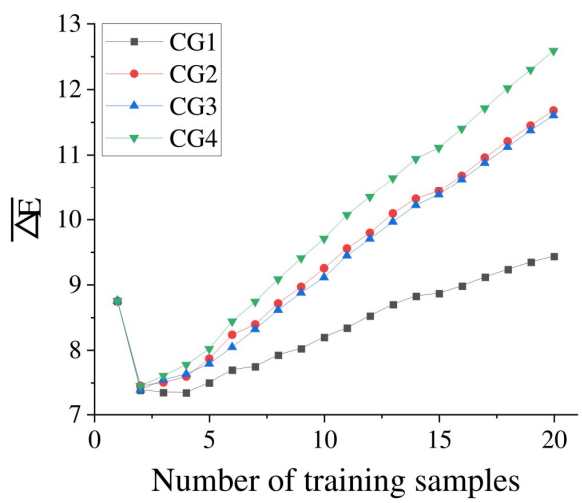

(c)

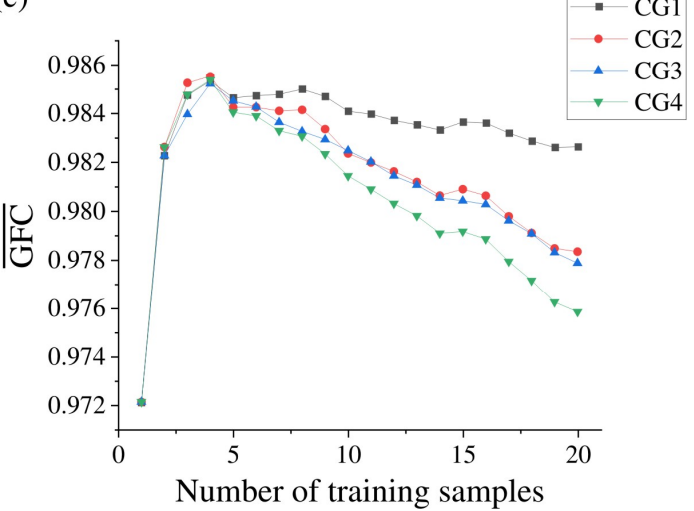

(b)

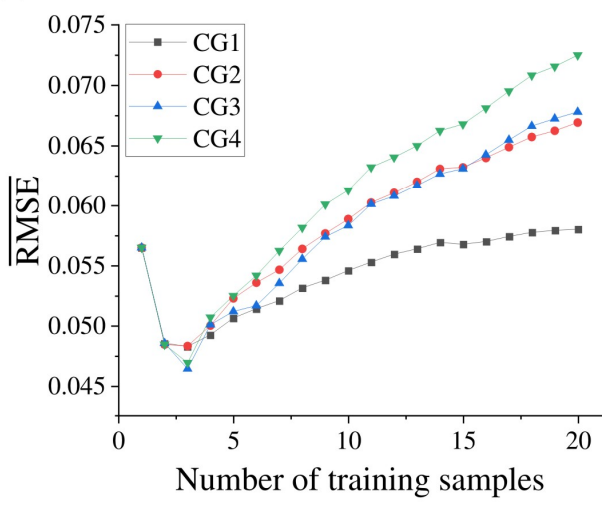

(d)

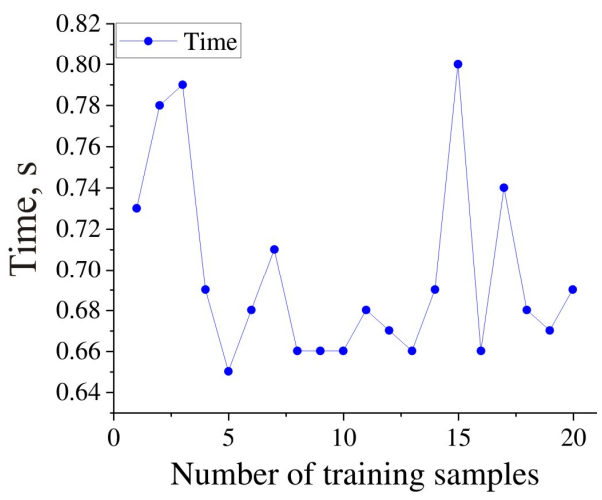

Fig. 3. Effect of sample number on the reconstruction accuracy of WSCG method: (a) the mean colour difference, (b) the mean RMSE, (c) the mean GFC, and (d) the calculation time. Curves CG1-CG4 correspond to the four models of WSCG described by Eqs. (7)-(10).

the $\overline{\Delta \mathrm{E}}$ value for the WSCG method reaches its minimum when the number of training samples is equal to 4 . When the number of training samples is equal to 3 , the $\overline{\mathrm{RMSE}}$ parameter is minimal (see Fig. 3b). GFC becomes maximal when the number of training samples is 4 (see Fig. 3c). Finally, the longest, the shortest and the average calculation times amount to $0.80 \mathrm{~s}, 0.65 \mathrm{~s}$ and 0.70 s, respectively (see Fig. $3 \mathrm{~d}$ ). Here the data concerns the number of training samples varying from 1 to 20. All the weighted models reveal high reconstruction accuracy at the numbers as small as $2-4$. The appropriate number of training samples for the WSCG method is equal to 3 .

One can draw the following conclusions from the results depicted in Fig. 3.

(1) Although there is no linear relationship among the colour difference, the RMSE and the GFC, the overall trends for the three evaluation indices are same. Therefore, we can improve further the spectral-matching degree (i.e., reduce the RMSE and increase the GFC) by reducing the colour difference of the reconstructed spectra.

(2) For the WSCG method, one can obtain the reconstructed spectra with a few training samples, so that the selected training samples correspond to large weights. When the number of training samples is greater than 4 , the reconstruction accuracy starts decreasing with further increase of the number. Therefore, only a few training samples with large weights can improve the spectral-reconstruction accuracy. Since the training samples with small weights are significantly different from the test samples, this can increase the reconstruction errors. 
(3) The WSCG method selects the same number of training samples for different testing samples, which simplifies calculations though increases the reconstruction error somewhat.

In the experiments with the WSCG-LOS method, based on the established smaller-colour difference group, we select the first hundred colour differences and the corresponding training samples to explore the relationship between the reconstruction accuracy and the number of local training samples. Fig. $4 \mathrm{a}, \mathrm{b}$ and $\mathrm{c}$ show the relationships between $\overline{\Delta \mathrm{E}}, \overline{\mathrm{RMSE}}, \overline{\mathrm{GFC}}$ and the number of local training samples. The $\overline{\Delta \mathrm{E}}$ and $\overline{\mathrm{RMSE}}$ parameters decrease gradually with increasing number of local training samples, while the $\overline{\mathrm{GFC}}$ parameter then gradually increases. When the number of local training samples is between 10 and $30, \overline{\Delta \mathrm{E}}$ and $\overline{\mathrm{RMSE}}$ are greatly reduced and $\overline{\mathrm{GFC}}$ is greatly increased. The improvement in the reconstruction accuracy is limited with further increase in the number of local training samples. Meanwhile, the calculation time increases gradually under the same conditions. Therefore, the appropriate number of the training samples for the WSCG-LOS method is 30. Then the selected local training samples correspond to large weights, which influence hard the spectral-reconstruction accuracy. For the most of testing samples, the reconstructed spectra with minimal colour difference can be obtained with a few local training samples. Only a small number of testing samples needs a large number of training samples to obtain the reconstructed spectra with minimal colour differences.

(a)

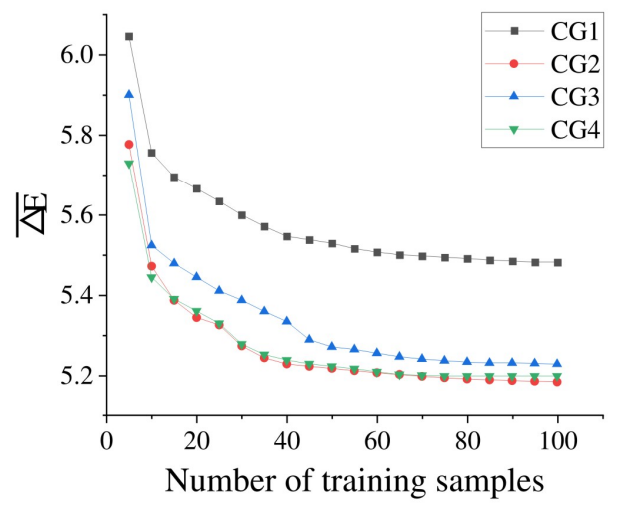

(c)

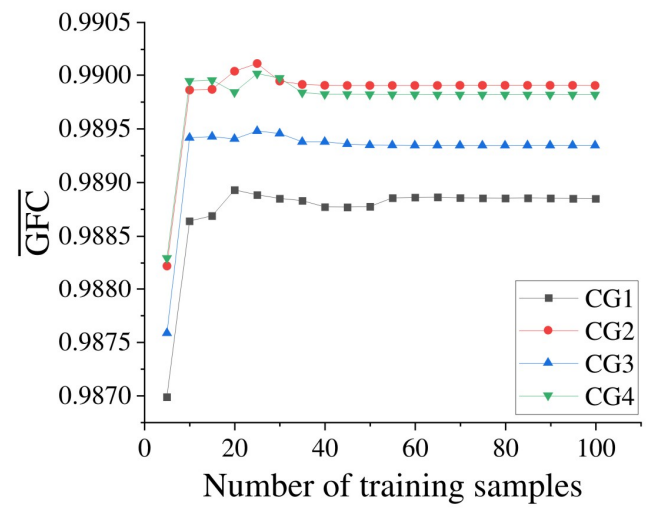

(b)

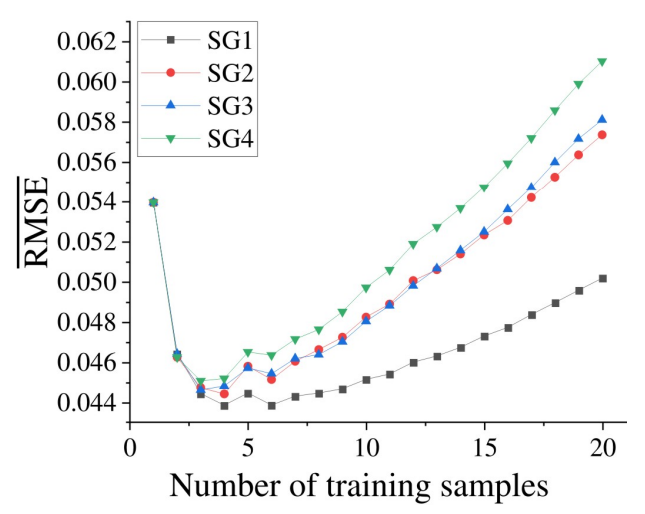

(d)

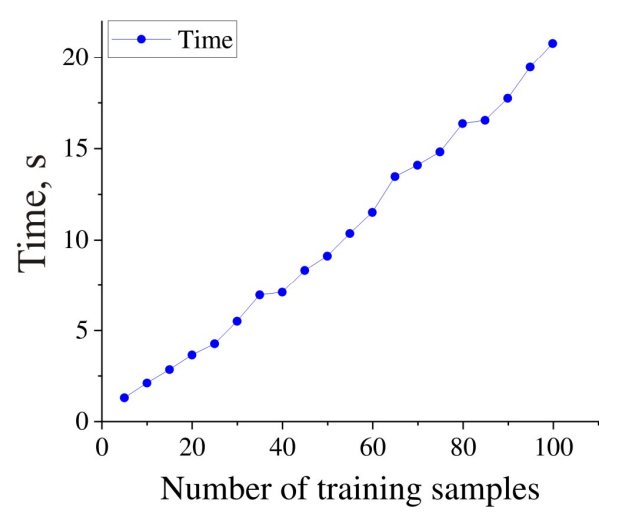

Fig. 4. Effect of sample number on the reconstruction accuracy of WSCG-LOS method: (a) the mean colour difference, (b) the mean RMSE, (c) the mean GFC, and (d) the calculation time. Curves CG1-CG4 correspond to the four models of WSCG described by Eqs. (7)-(10).

Ukr. J. Phys. Opt. 2020, Volume 21, Issue 2 
In our experiments, we have used the PINV, WE, WSCG, WSCG-GOS and WSCG-LOS methods to reconstruct the spectral reflectance of SG140. The colour difference, the RMSE and the GFC have been used when evaluating the reconstruction accuracy. The reconstruction accuracy and the calculation time of the above methods are compared in Table 1. Under interference of the system noise, the reconstruction accuracy of the PINV method is the worst. The WE method shows good enough performance concerned with the standard deviation of the evaluation index. This indicates that this approach has the highest data stability. The WSCG method takes much less calculation times than the other methods but its reconstruction accuracy is poor. The reconstruction accuracy of the WSCG-GOS method is the highest at the cost of the longest calculation time. Finally, the WSCG-LOS method can reconstruct the spectra with high enough accuracy in a short time.

Table 1. Reconstruction accuracies found for different methods: CG1-CG4 correspond to four different models of the WSCG method, which are described by Eqs. (7)-(10). The notation 'Std' implies 'standard deviation'.

\begin{tabular}{ccccccccc}
\hline \multirow{2}{*}{ Method } & \multicolumn{3}{c}{$\Delta \mathrm{E}$} & \multicolumn{2}{c}{ RMSE } & \multicolumn{2}{c}{ GFC } & \multirow{2}{*}{ Time, s } \\
\cline { 3 - 8 } & & Mean & Std & Mean & Std & Mean & Std & \\
\hline \multirow{4}{*}{ WSCG } & CG1 & 7.3491 & 6.9050 & 0.0483 & 0.0504 & 0.9847 & 0.0432 & 0.40 \\
& CG2 & 7.4974 & 7.0693 & 0.0483 & 0.0506 & 0.9853 & 0.0400 & 0.38 \\
& CG3 & 7.5391 & 7.2687 & 0.0464 & 0.0502 & 0.9840 & 0.0439 & 0.40 \\
& CG4 & 7.5985 & 7.3285 & 0.0469 & 0.0499 & 0.9848 & 0.0405 & 0.39 \\
& CG1 & 5.4803 & 5.8082 & 0.0405 & 0.0460 & 0.9880 & 0.0321 & 9.18 \\
WSCG- & CG2 & 5.1738 & 5.6376 & 0.0362 & 0.0388 & 0.9899 & 0.0280 & 8.57 \\
GOS & CG3 & 5.2239 & 5.7957 & 0.0349 & 0.0389 & 0.9893 & 0.0296 & 9.87 \\
& CG4 & 5.1978 & 5.7134 & 0.0358 & 0.0384 & 0.9898 & 0.0273 & 9.26 \\
& CG1 & 5.5993 & 5.8648 & 0.0408 & 0.0464 & 0.9889 & 0.0323 & 1.57 \\
WSCG- & CG2 & 5.2729 & 5.6926 & 0.0378 & 0.0425 & 0.9889 & 0.0280 & 1.37 \\
LOS & CG3 & 5.3868 & 5.8188 & 0.0368 & 0.0418 & 0.9895 & 0.0298 & 1.65 \\
& CG4 & 5.2776 & 5.7237 & 0.0371 & 0.0404 & 0.9900 & 0.0273 & 1.47 \\
WE & & 5.8789 & 3.8970 & 0.0417 & 0.0336 & 0.9905 & 0.0237 & 1.85 \\
PINV & 16.938 & 6.3916 & 0.1294 & 0.0717 & 0.9852 & 0.0191 & 1.68 \\
\hline
\end{tabular}

Let us analyze the experimental results depicted in Fig. 3, Fig. 4 and Table 1.

(1) The WSCG method selects the same number of training samples for all the testing samples, so that this method needs a very little calculation time. However, the appropriate method of sample selection leads to large reconstruction errors.

(2) For the WSCG-LOS method, the reconstruction accuracy and the calculation time increase gradually with increasing number of local training samples. When the number of training samples reaches 139, this method becomes equivalent to the WSCG-GOS, and its reconstruction accuracy can reach a maximum. However, the calculation time can increase greatly with increasing number of local training samples. Only a small number of testing samples needs a large number of training samples to obtain the reconstructed spectra with a minimal colour difference. Therefore, in spite of the improved reconstruction accuracy, the increased calculation time of the WSCG-GOS method is unacceptable in any practical situation. In this respect, the WSCG-LOS method can achieve a good balance between the reconstruction accuracy and the calculation time. 
(3) At the same time, the WSCG-LOS method selects the colour differences as weights. Therefore, there is less improvement in the spectral-matching degree if compared with the WE method. Then the metamerism phenomenon still persists.

\subsection{Comparison of WSREG, WSREG-GOS and WSREG-LOS methods}

We have reproduced the WSREG method basing on the experimental procedures typical for the WSCG method [17]. The main regularities, which are observed for the $\overline{\Delta \mathrm{E}}, \overline{\mathrm{RMSE}}$ and $\overline{\mathrm{GFC}}$ parameters with changing number of training samples (in the interval 1-20), are shown in Fig. 5. As seen from Fig. 5a, the $\overline{\Delta \mathrm{E}}$ parameter of the WSREG method reaches its minimum when the number of training samples becomes equal to 3 . When the number of training samples is 3 or 4 , the $\overline{\text { RMSE }}$ for the WSREG method reaches a minimum (see Fig. 5b). The $\overline{\text { GFC }}$ parameter for the WSREG method becomes maximal when the number of training samples is 3 (see Fig. 5c). The longest, the shortest and the average calculation times amount respectively to $0.79 \mathrm{~s}, 0.53 \mathrm{~s}$ and 0.71 s (see Fig. 3d). All the weighting models manifest high enough reconstructed accuracy at the number of training samples equal to 2-4. The appropriate number of training samples is 3 in the case of WSREG method.

(a)

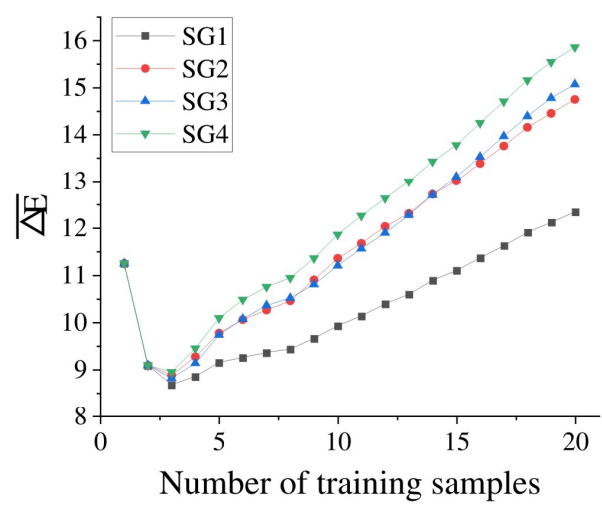

(c)

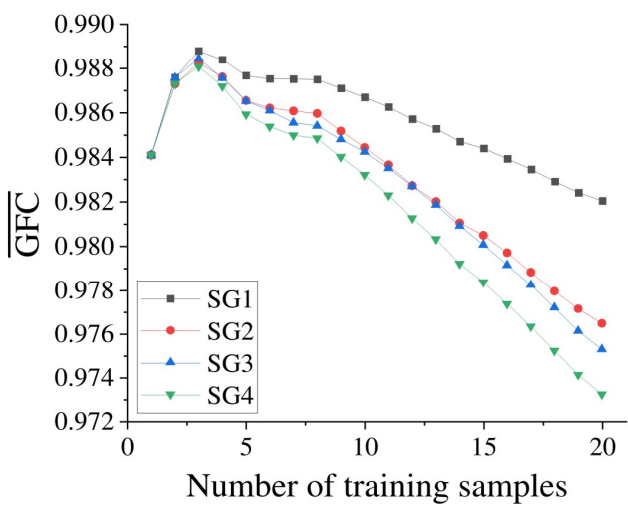

(b)

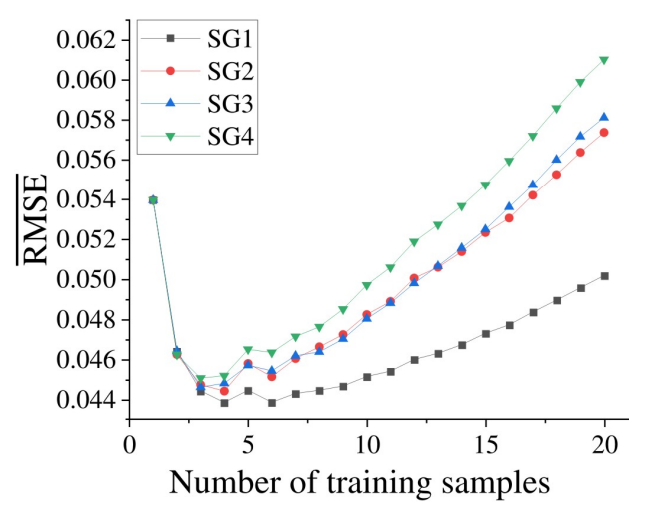

(d)

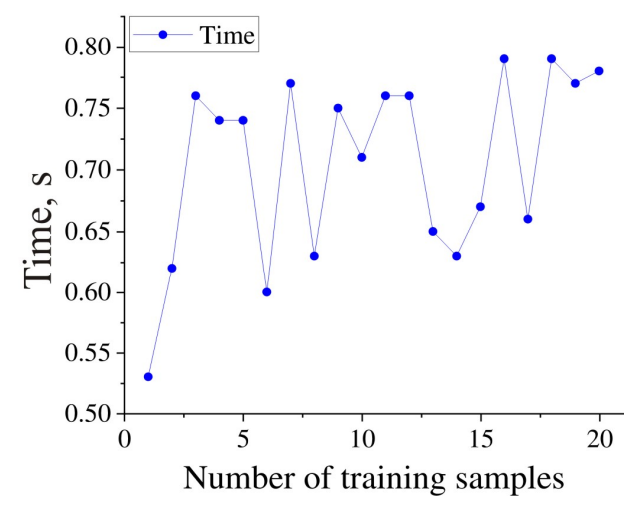

Fig. 5. Effect of sample number on the reconstruction accuracy of WSREG method: (a) the mean colour difference, (b) the mean RMSE, (c) the mean GFC, and (d) the calculation time. Curves CG1-CG4 correspond to the four models of WSREG described by Eqs. (12)-(15).

The following conclusions become evident from the data presented in Fig. 5.

(1) One can obtain the spectra reconstructed with the WSREG method using a small number of

Ukr. J. Phys. Opt. 2020, Volume 21, Issue 2 
training samples. Here the selected training samples correspond to larger weights. These samples with large weights can improve the reconstruction accuracy. On the other hand, the training samples with smaller weights are significantly different from the testing sample, which can reduce the reconstruction accuracy.

(2) The WSREG method selects the same number of training samples for different testing samples, which simplifies calculations.

However, the same selected number of training samples does not represent an optimal solution in the most of cases, which increases the reconstruction error to some extent.

In our experiments with the WSREG-LOS method, which are based on the smaller-SRE group, we select the first hundred colour differences and the corresponding training samples to explore the relationship between the reconstruction accuracy and the number of training samples. Fig. $6 \mathrm{a}, \mathrm{b}$ and $\mathrm{c}$ show the dependences of $\overline{\mathrm{\Delta E}}, \overline{\mathrm{RMSE}}$ and $\overline{\mathrm{GFC}}$ on the number of training samples. With increasing number of local training samples, the $\overline{\Delta \mathrm{E}}$ and $\overline{\mathrm{RMSE}}$ parameters decrease and $\overline{\mathrm{GFC}}$ increases. When the number of training samples is somewhere between 10 and 40 , the $\overline{\Delta \mathrm{E}}$ and $\overline{\mathrm{RMSE}}$ parameters become greatly reduced and the $\overline{\mathrm{GFC}}$ is greatly increased.

(a)

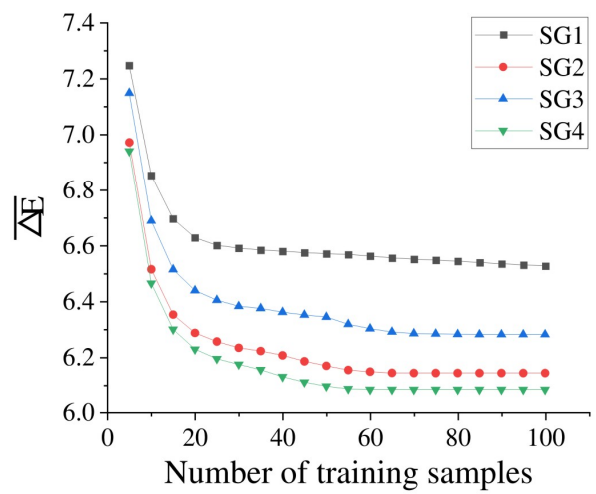

(c)

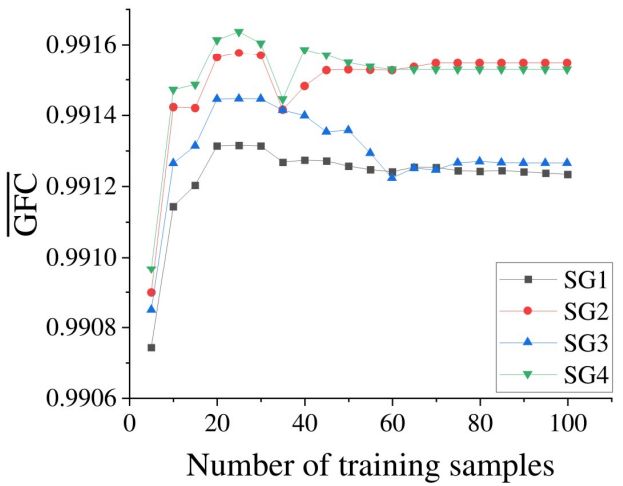

(b)

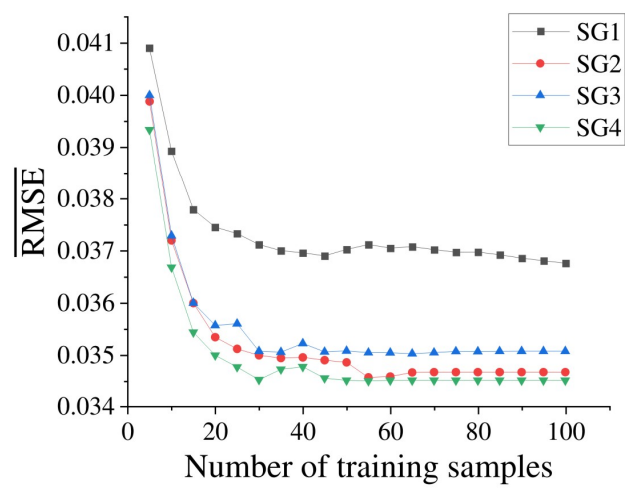

(d)

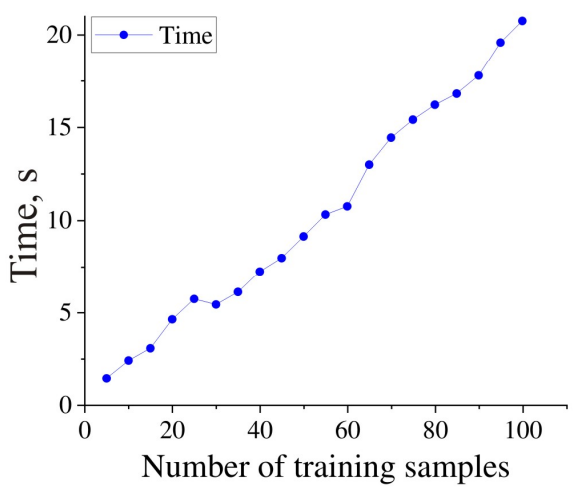

Fig. 6. Effect of sample number on the reconstruction accuracy of WSREG-LOS method: (a) the mean colour difference, (b) the mean RMSE, (c) the mean GFC, and (d) the calculation time. Curves CG1-CG4 correspond to the four models of WSREG described by Eqs. (12)-(15).

With further increase in the number of local training samples, the improvement of the reconstruction accuracy is rather limited. On the other hand, then the calculation time increases gradually. Therefore, the optimal number of training samples for the WSREG-LOS method is 30. 
The selected local training samples with large weights affect notably the spectral-reconstruction accuracy. Most of the testing samples obtain the reconstructed spectra with a minimal colour difference. This needs only a small number of local training samples.

We have used the WSREG, WSREG-GOS and WSREG-LOS methods to reconstruct the spectral reflectance of SG140. The colour difference, along with the RMSE and GFC parameters, has been selected to evaluate the reconstruction accuracy. The reconstruction accuracy and the calculation time of the above methods are illustrated in Table 2. The accuracy for the WSREGGOS method is the highest and the calculation time for the WSREG-GOS method is the longest. On the other hand, the reconstruction accuracy for the WSREG method is the worst and its calculation time is the shortest. When compared with the WSREG and WSREG-GOS methods, the WSREG-LOS can reconstruct the spectra with high enough accuracy in a short time.

Table 2. Reconstruction accuracies for different methods: SG1-SG4 correspond to four different models of the WSREG method, which are described by Eqs. (12)-(15). Notation 'Std' implies 'standard deviation'.

\begin{tabular}{ccccccccc}
\hline \multirow{2}{*}{ Method } & \multicolumn{2}{c}{$\Delta \mathrm{E}$} & \multicolumn{2}{c}{ RMSE } & \multicolumn{2}{c}{ GFC } & Time, \\
\cline { 3 - 8 } & & Mean & Std & Mean & Std & Mean & Std & s \\
\cline { 3 - 8 } WSREG & SG1 & 8.6726 & 7.3891 & 0.0444 & 0.0392 & 0.9888 & 0.0244 & 0.33 \\
& SG2 & 8.8733 & 7.5017 & 0.0447 & 0.0396 & 0.9882 & 0.0259 & 0.34 \\
& SG3 & 8.8094 & 7.6334 & 0.0446 & 0.0401 & 0.9884 & 0.0255 & 0.34 \\
& SG4 & 8.9418 & 7.6422 & 0.0451 & 0.0402 & 0.9881 & 0.0266 & 0.35 \\
WSREG- & SG1 & 6.5078 & 6.5938 & 0.0364 & 0.0345 & 0.9912 & 0.0191 & 9.89 \\
GOS & SG2 & 6.1424 & 6.3929 & 0.0347 & 0.0324 & 0.9915 & 0.0185 & 8.64 \\
& SG3 & 6.2807 & 6.6046 & 0.0351 & 0.0339 & 0.9913 & 0.0194 & 9.79 \\
& SG4 & 6.0832 & 6.4557 & 0.0345 & 0.0331 & 0.9915 & 0.0186 & 9.52 \\
WSREG- & SG1 & 6.5909 & 6.5819 & 0.0371 & 0.0352 & 0.9913 & 0.0190 & 1.44 \\
LOS & SG2 & 6.2328 & 6.3990 & 0.0350 & 0.0324 & 0.9916 & 0.0185 & 1.31 \\
& SG3 & 6.3821 & 6.5931 & 0.0351 & 0.0337 & 0.9914 & 0.0192 & 1.57 \\
WE & SG4 & 6.1735 & 6.4536 & 0.0345 & 0.0330 & 0.9916 & 0.0185 & 1.41 \\
PINV & & 5.8789 & 3.8970 & 0.0417 & 0.0336 & 0.9905 & 0.0237 & 1.85 \\
& & 16.938 & 6.3916 & 0.1294 & 0.0717 & 0.9852 & 0.0191 & 1.68 \\
\hline
\end{tabular}

The following conclusions can be drawn from the experimental results depicted in Fig. 5, Fig. 6 and Table 2.

(1) The WSREG method selects the same number of training samples for the testing dataset. Then the method needs a very little calculation time, although the reconstruction errors are large.

(2) The reconstruction accuracy and the calculation time of the WSREG-LOS method increase with increasing number of local training samples. When this number reaches 139, the WSREG-LOS method becomes equivalent to the WSREG-GOS. The reconstruction accuracy can reach a maximum but then the calculation time increases greatly. Only a small number of testing samples needs a large number of training samples in order to obtain the reconstructed spectra with a minimal colour difference. Therefore the increased calculation time peculiar for the WSREG-GOS method is unacceptable in practice, in spite of the improved reconstruction accuracy. On the other hand, the WSREG-LOS method can balance satisfactorily the reconstruction accuracy and the calculation time.

(3) The WSREG-LOS method selects the SREs as weights. The smaller-SRE group can be obtained by calculating the SREs between the first reconstructed spectrum of a single testing 
sample and the original spectral reflectance of a training dataset. We use the WE method to obtain the first reconstructed spectrum. Unfortunately, it manifests some reconstruction errors, which reduce the accuracy of the WSREG-LOS method. Therefore the spectral-matching degree of the WSREG-LOS method is improved to a certain extent, when compared with the WSCG-LOS and WE methods. Nonetheless, the colour difference for the WSREG-LOS method is greater than that of the WSCG-LOS.

(4) The WE method reconstructs the spectral reflectance of the testing dataset using a training dataset to construct the conversion matrix. Therefore, the WE method has better data stability. For the WSCG and WSREG methods, the reconstructed spectra of the testing dataset depend on the colour difference or the SRE and the corresponding spectral reflectance of the training dataset. As a result, the data stability for the WSCG and WSREG methods is rather poor.

\subsection{Effect of weight on the colour difference}

According to Table 1 and Table 2, we have selected the CG4 model for the WSCG-LOS and WE methods, and the SG4 model for the WSREG-LOS method. This has been used to obtain the reconstructed spectra R1, R2 and R3, respectively. The MW method has been employed to obtain the spectral estimation with higher accuracy. In our experiments, the weights taken for different methods can be selected by minimizing the colour difference between the original and reconstructed spectral reflectances. As seen from the histogram shown in Fig. 7, the orange, green and purple regions represent respectively the weights of the WSCG-LOS, WSREG-LOS and WE methods. The area of the orange region is the largest, and so the weight of the WSCG-LOS method is. In other words, the WSCG-LOS method is the most important in accomplishing the MW method.

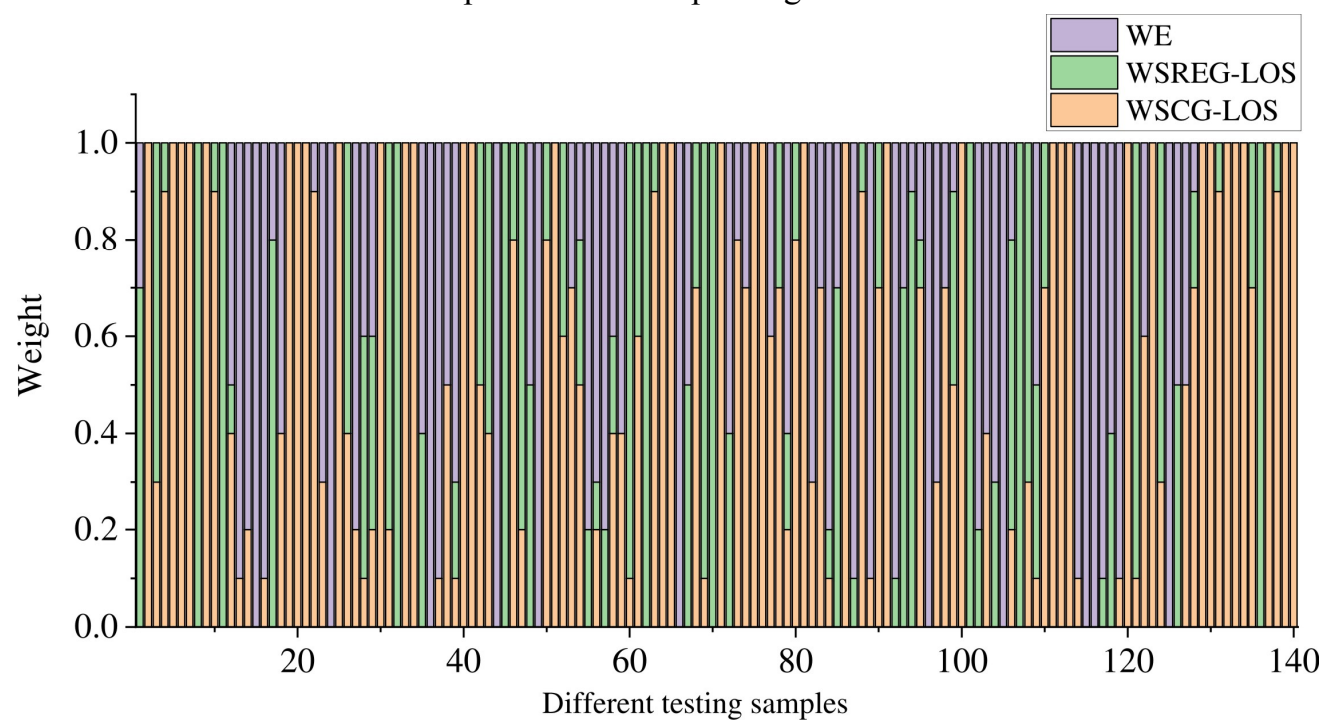

Fig. 7. Weights of different methods.

In order to explore the influence of different weights of the WSCG-LOS method on the colour difference, the $10^{\text {th }}$ colour block of the SG140 model has been selected. Different weights have been multiplied by the spectral estimation of the WSCG-LOS method. As seen from Fig. 8, the colour difference decreases noticeably with increasing weight of the WSCG-LOS method. It reaches a minimum when the weight is equal to 0.9 . Moreover, when the weight of the WSCGLOS method varies from 0 to 1 , the colour difference is reduced to about 6.2. Issuing from this fact, we conclude that the WSCG-LOS method has the greatest impact on the overall 
reconstruction accuracy. The best way to get the spectral estimation with higher accuracy is to find the appropriate weight of the WSCG-LOS method and combine the other two methods for obtaining the minimal colour difference.

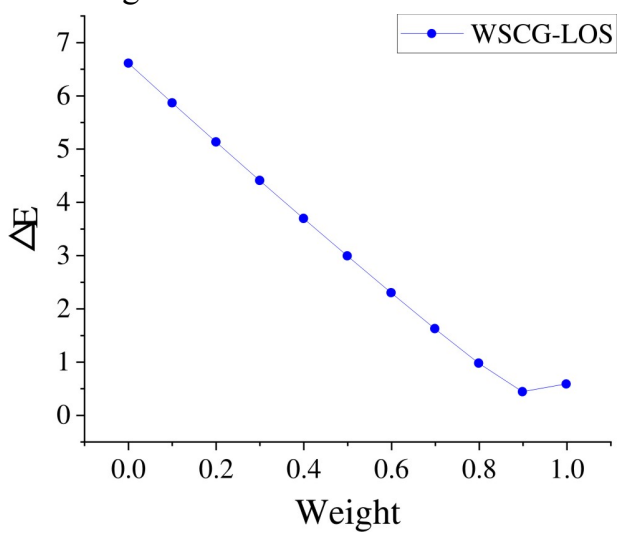

Fig. 8. Effect of weight of the WSCG-LOS method on the colour difference.

\subsection{Accuracy comparison for the reconstructed spectra}

In order to reconstruct the spectral reflectance of SG140, we have used the WE method, the CG4 model for the WSCG-LOS method, the SG4 model for the WSREG-LOS and the CG4+SG4+WE model for the MW method. The original and reconstructed spectral curves obtained with different methods are shown in Fig. 9. The colour difference, the RMSE and the GFC have been used to evaluate the reconstruction accuracy (see Table 3).
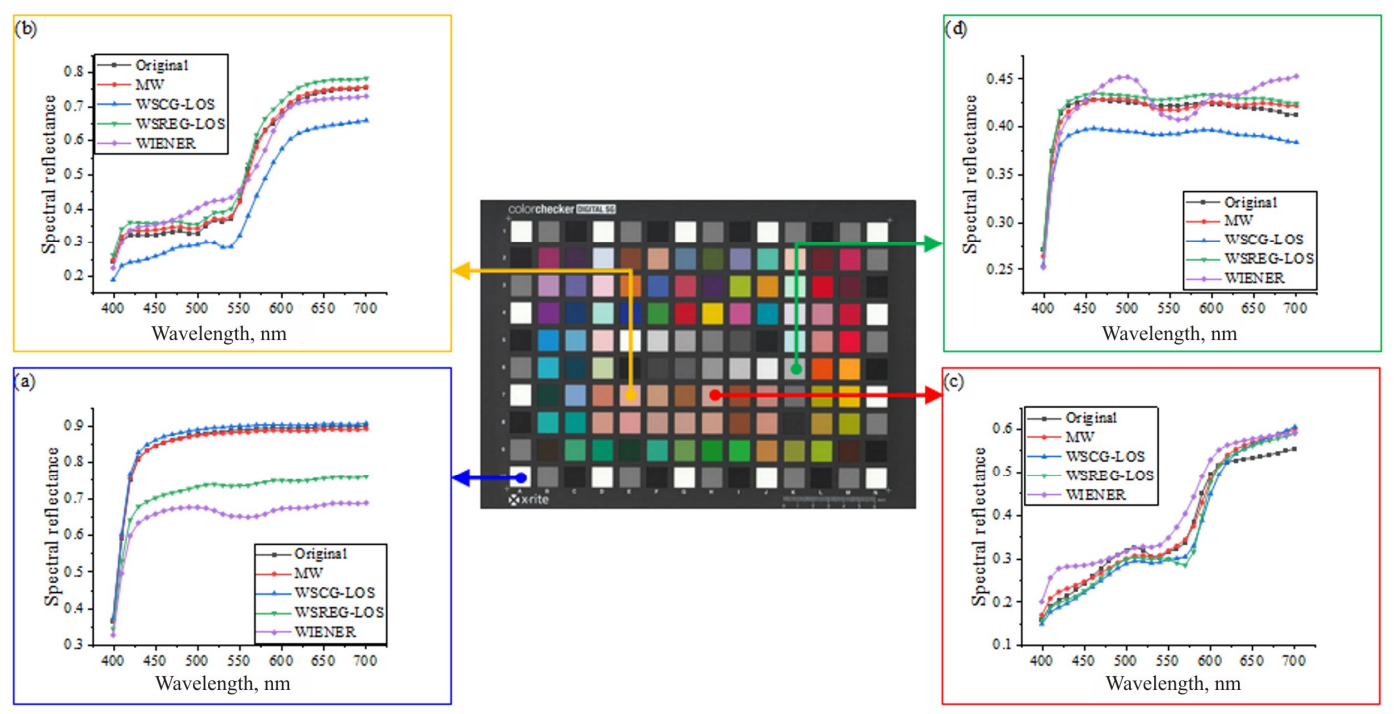

Fig. 9. Examples of reconstructed spectral curves: $10^{\text {th }}(a), 47^{\text {th }}(\mathrm{b}), 77^{\text {th }}(\mathrm{c})$ and $106^{\text {th }}$ (d) colour blocks of SG140.

As seen from Fig. 9, the degree of spectral match between the original and reconstructed spectra, which are obtained with the MW method, is higher than that of the other methods. Therefore the reconstruction accuracy of the MW method is the highest. One can see from Table 3 that the $\overline{\Delta \mathrm{E}}$ and $\overline{\mathrm{RMSE}}$ parameters for the MW method are the smallest, while its $\overline{\mathrm{GFC}}$ is the largest. Moreover, the accuracy of this method is the highest. The standard deviation of the colour difference, the RMSE and the GFC are the smallest for the MW method, which indicates that the MW method reveals the highest data stability. Therefore, we state that the MW method improves both the reconstruction accuracy and the data stability. The calculation time needed for 
reconstructing the SG140 model basing on the MW method is $2.35 \mathrm{~s}$. Keeping in mind its improved reconstruction accuracy, one can conclude that the increase in the calculation time for the MW method is quite acceptable in practice.

Table 3. Comparison of reconstruction accuracies typical for different methods.

\begin{tabular}{cccccccc}
\hline \multirow{2}{*}{ Method } & \multicolumn{2}{c}{$\Delta \mathrm{E}$} & \multicolumn{2}{c}{ RMSE } & \multicolumn{2}{c}{ GFC } & \multirow{2}{*}{ Time, s } \\
\cline { 2 - 7 } & Mean & Std & Mean & Mean & Std & Mean & \\
\hline MW & 2.6685 & 3.1366 & 0.0225 & 0.0232 & 0.9946 & 0.0141 & 2.35 \\
WSCG-LOS & 5.2776 & 5.7237 & 0.0371 & 0.0404 & 0.9900 & 0.0273 & 1.47 \\
WSREG-LOS & 6.1735 & 6.4536 & 0.0345 & 0.0330 & 0.9916 & 0.0185 & 1.41 \\
WE & 5.8789 & 3.8970 & 0.0417 & 0.0336 & 0.9905 & 0.0237 & 1.85 \\
PINV & 16.938 & 6.3916 & 0.1294 & 0.0717 & 0.9852 & 0.0191 & 1.68 \\
\hline
\end{tabular}

\section{Discussion and conclusion}

To overcome shortcomings of the WSCG method, we have proposed its modification, the WSCGGOS approach. If compared with the canonical WSCG version, the WSCG-GOS method selects the appropriate number of training samples for different testing samples by traversing the training sample set and minimizing colour difference. This leads to improved reconstruction accuracy. However, in spite of this fact, the increased calculation time of the WSCG-GOS method is unacceptable in practice. Therefore we have built the WSCG-LOS method which achieves a good balance between the reconstruction accuracy and the calculation time. The WSCG-LOS selects a small number of local training samples for different testing samples, and the number of selected training samples is the same. In the local space of training sample sets, we select the appropriate number of training samples for different testing samples by minimizing their colour difference. This solution reduces greatly the calculation time and still is characterized with high enough reconstruction accuracy.

Since the colour difference is taken as a weight, the metamerism phenomenon still exists within the WSCG-LOS method. Concerning the WSCG method, we have suggested the WSREG method to obtain the spectral estimation with a higher spectral-matching degree. We have also explored the advantages and disadvantages of the WSREG, WSREG-GOS and WSREG-LOS methods. The WSREG-LOS method can also achieve a good balance between the reconstruction accuracy and the calculation time. However, the colour difference typical for this method is greater than that of the WSCG-LOS. The reasons are as follows. We obtain the SRE group through calculating the SRE between the first reconstructed spectrum of a single testing sample and the original spectral reflectance of a training dataset, whereas the WE method is used for obtaining the first reconstructed spectra of testing dataset. Since the WE method has a certain degree of reconstruction error, the reconstruction accuracy of the WSREG-LOS method is reduces. When compared with the WE method, the data stability peculiar for the WSCG-LOS and WSREG-LOS methods is poor.

The MW method weights the spectra reconstructed by different methods to obtain the spectral estimation with a minimum colour difference. This boosts the reconstruction accuracy and overcomes the shortcomings of those methods. Our experimental results indicate that the MW method improves both the reconstruction accuracy and the data stability. Moreover, the MW method reduces the metamerism phenomenon and the reconstructed spectral reflectance has no negative values. The experimental data shows that the method suggested by us is feasible both in theoretical and technologic aspects. The spectral-reflectance reconstruction method based on the 
MW is universal in some sense. In practice, we can choose the spectral-reconstruction methods with higher accuracy and greater calculation speed in order to improve the reconstruction accuracy of the MW method.

\section{Acknowledgements}

The authors wish to appreciate valuable comments and suggestions on our manuscript from the editor and anonymous reviewers.

\section{References}

1. Xiao K D, Zhu Y, Li C, Connah D, Yates J M and Wuerger S, 2016. Improved method for skin reflectance reconstruction from camera images. Opt. Express. 24: 14934-14950.

2. Liang J X, Xiao K D, Pointer M R, Wan X and Li C, 2019. Spectra estimation from raw camera responses based on adaptive local-weighted linear regression. Opt. Express. 27: $5165-5180$.

3. Li J X, Liu W J, Li T, Rozen I, Zhao J, Bahari B, Kante B and Wang J, 2016. Swimming micro-robot optical nanoscopy. Nano Lett. 16: 6604-6609.

4. Wang H W, Li J and Chen G X, 2011. Study on key issues of multi-spectral color reproduction technique. Adv. Mater. Res. 117: 93-96.

5. Zhu Y H, Li B and Xu X Y, 2012. Spectral reconstruction and accuracy appraisal based on pseudo inverse method. IEEE Symposium on Photonics and Optoelectronics, Shanghai, 1-3

6. Yuasa T, Honma R, Funamizu H, Nishidate I and Aizu Y, 2014. Color adjustment algorithm adapted to the spectral reflectance estimation method. Opt. Rev. 21: 369-372.

7. Zhang X D, Wang Q, Yang G F and Wang M, 2014. Acquiring multi-spectral images by digital still cameras based on XYZLMS interim connection space. Chin. Opt. Lett. 12: 113302.

8. Zhu Y H, Li B, Chen Q and He S H, 2013. Two spectral reconstruction methods and their accuracy comparison. Appl. Mech. Mater. 239-240: 140-144.

9. Zhang Y and Zhou S S, 2013. Research on reconstruction of spectral reflectance based on principal component analysis. Appl. Mech. Mater. 262: 53-58.

10. Agahian F, Amirshahi S A and Amirshahi S H, 2008. Reconstruction of reflectance spectra using weighted principal component analysis. Color Res. Appl. 33: 360-371.

11. Yamaguchi M, Ohyama N and Murakami Y, 2009. Piecewise Wiener estimation for reconstruction of spectral reflectance image by multipoint spectral measurements. Appl. Opt. 48: 2188-2202.

12. Nishidate I, Maeda T, Niizeki K and Aizu Y, 2013. Estimation of melanin and hemoglobin using spectral reflectance images reconstructed from a digital RGB image by the Wiener estimation method. Sensors. 13: 7902-7915.

13. Zhang L H, Liang D, Li B, Kang Y, Pan Z, Zhang D and Ma X, 2016. Study on the key technology of spectral reflectivity reconstruction based on sparse prior by a single-pixel detector. Photon. Res. 4: 115-121.

14. Li B, Zhang H J, Zhang L H, Kang Y, Zhan W, Yi W and Zhang D, 2017. Study on the key technology of spectral reflectance reconstruction based on a single pixel detector. Laser Phys. Lett. 14: 125203.

15. Liang $\mathrm{J} X$ and Wan $X X, 2017$. Optimized method for spectral reflectance reconstruction from camera responses. Opt. Express. 25: 28273-28287.

16. Ostu H, Yamamoto $M$ and Hachisuka T, 2018. Reproducing spectral reflectances from tristimulus colours. Comp. Graph. Forum, 37: 370-381. 
17. Cao B, Liao N F and Cheng H B, 2017. Spectral reflectance reconstruction from RGB images based on weighting smaller color difference group. Color Res. Appl. 42: 327-332.

18. Zhang X D, Wang Q, Li J C, Zhou X, Yang Y and Xu H, 2016. Estimating spectral reflectance from camera responses based on CIE XYZ tristimulus values under multiilluminants. Color Res. Appl. 42: 68-77.

19. Kong L J, Zeng X, Zhang L H, Zhan W J, Zeng W C, 2019. Research on spectral reflectance reconstruction based on genetic algorithm for selecting multi-illuminants. Spectroscopy and Spectral Analysis. 39: 1162-1168.

20. Heikkinen V, 2018. Spectral reflectance estimation using Gaussian processes and combination kernels. IEEE Transactions on Image Processing. 27: 3358-3373.

21. Koundinya S, Sharma H, Sharma M, Upadhyay A, Manekar R, Mukhopadhyay R, Karmakar A and Chaudhury S, 2018. 2D-3D CNN based architectures for spectral reconstruction from RGB Images. IEEE CVF Conference on Computer Vision and Pattern Recognition Workshop, p. 957-954.

22. Han X H, Shi B X and Zheng Y Q, 2018. Residual HSRCNN: Residual hyper-spectral reconstruction CNN from an RGB image. $24^{\text {th }}$ IEEE Int. Conf. on Patterns Reconstruction, p. 2664-2669.

23. Shimano $\mathrm{N}$ and Hironaga M, 2006. Recovery of spectral reflectances of objects being imaged without prior knowledge. IEEE Transactions on Image Processing. 15: 1848-1856.

24. Zhang L H, Li B, Liang D and Ma X H, 2016. Study on the key technology of spectral reflectance reconstruction based on the weighted measurement matrix. Laser Phys. 26: 075202

25. Yoo J H, Kyung W J, Ha H G and Ha Y-H, 2013. Estimation of reflectance based on properties of selective spectrum with adaptive wiener estimation. Proc. SPIE. 8652: 86520D.

26. Wu G, Liu Z and Zhang J, 2015. Spectral color reproduction from CIE tristimulus values using a node address array selection technique. Sign. Proc. Image Proc. \& Patt. Recogn. 8: $1786-1790$.

27. Song J H, Kim C and Yoo Y, 2015. Vein visualization using a smart phone with multispectral Wiener estimation for point-of-care applications. IEEE J. Biomed. Health Inform. 19: 733788.

28. Zhang L H, Li B and Pan Z L, Liang D, Kang Y, Zhang D and Ma X, 2016. A method for selecting training samples based on camera response. Laser Phys. Lett. 13: 095201.

29. Cao B, Liao N F, Li Y S and Cheng H, 2016. Improving reflectance reconstruction from tristimulus values by adaptively combining colorimetric and reflectance similarities. Opt. Eng. 56: 053104

30. Niu J, Zhang X D and Wang Q, 2012. Study on the color representing accuracy of spectrum with different wavelength range and interval. Appl. Mech. Mater. 262: 13-17.

Leihong Zhang, Runchu Xu, Shuangquan Lu, Liuhua Yang, Xiao Yuan, Kaiming Wang and Dawei Zhang. 2020. Reconstruction of spectral reflectance based on mixed weighting and local optimization. Ukr.J.Phys.Opt. 21: 65 - 83 doi: 10.3116/16091833/21/2/65/2020

Анотація. Високоточне відтворення кольорів можна здійснити за допомогою реконструкиіі спектрального відбивання поверхні предмета. Конкретні галузі, які потребують такого відтворення, варіюються від кольорового друку $і$ аж до художнього мистеитва. Для підвищення точності реконструкиї спектрального відбивання нами запропоновано метод реконструкиіі спектрального відбивання на основі змішаного зважування (33). Цей метод є поєднанням низки більш ранніх методів, а саме методу 
оцінки Вінера $і$ так званих методів зважування по групі з меншою різницею кольорів $i$ зважування по групі з меншою похибкою спектрального відбивання. Зокрема, одержано спектральну оцінку з вищою точністю реконструкції шляхом зважування реконструйованих спектрів, отриманих за різними методами. Ваги ичих методів було обрано шляхом мінімізації різниці кольорів. Метод 33 повністю використовує переваги методів, які лежать в його основі. Він володіє високою точністю та нівелює недоліки згаданих методів. Результати наших експериментів підтверджують, щзо метод 33 підвищує точність реконструкиії та стабільність даних для спектрального відбивання. 